\title{
La Pediatría de Atención Primaria en Andalucía: análisis de la situación y propuestas de futuro
}

\author{
C. Valdivia Jiménez ${ }^{\mathrm{a}}$, N. Palomino Urda \\ aPresidente de APap-Andalucía. Pediatra. CS Los Boliches. Fuengirola, Málaga. España. \\ bVicepresidenta de APap-Andalucía. Pediatra. CS Las Flores. Granada. España.
}

\begin{abstract}
Resumen
Los pediatras que trabajamos en el primer nivel asistencial estamos de acuerdo con los expertos que los países con una Atención Primaria (AP) de calidad, presentan mejores índices de salud, son más eficientes y reparten de forma más equitativa los recursos sanitarios entre la población.

La Pediatría de AP (PAP) en nuestra Comunidad Autónoma tiene en este momento déficit de profesionales, que es más acusado en las zonas rurales. Esto supone una amenaza para el actual modelo de asistencia infantil, relegando a un segundo plano aspectos tan importantes como son la calidad de la asistencia pediátrica, las expectativas de las familias y las opiniones de los profesionales sanitarios.

El pediatra se ha visto aislado dentro de los Equipos de Atención Primaria y apenas ha recibido apoyo del resto de profesionales del mismo. Es necesario que se consigan "equipos de profesionales" que compartan una población, en un entorno geográfico delimitado, que la estructura y organización de los centros de salud posibilite espacios temporales y físicos para el trabajo en equipo y que se avance en fórmulas más actuales de gestión de la demanda, que impliquen a cada uno de los miembros del equipo en la respuesta a las demandas de la ciudadanía.

La PAP debe orientar su práctica hacia la calidad asistencial y en la consecución de objetivos. Se deben establecer criterios e indicadores de calidad y de buenas prácticas.

Palabras clave: Pediatría. Atención Primaria. Trabajo en equipo. Calidad.

The Primary Care Pediatrics in Andalusia: situation analysis and proposals for the future

Abstract

The pediatricians who work in the first level of care agree with experts that countries with a high quality Primary Health Care (AP), have better health rates, are more efficient and distribute health resources more equitably among the population.

Primary Care Pediatrics in our region has at present a shortage of professionals, which is more pronounced in rural areas. This poses a threat to the current model of child care, relegating to the background such important issues as the quality of pediatric care, family expectations and the health professionals' opinions.
\end{abstract}

Carlos Valdivia Jiménez: cvaldivia@telefonica.net

Los autores declaran no presentar conflictos de intereses en relación con la preparación y publicación de este artículo. 
The pediatrician has been isolated within the Primary Care Teams and has hardly received support from other professionals of it. It is necessary to get "professional teams" that share a population in a defined geographical environment, that the structure and organization of health centers enable temporal and physical spaces for team work and that progress on current formulas of demand management are made, involving each of the team members in responding to the demands of citizenship.

The AP Pediatrics should aim its practice to the quality of care and to the achieving of goals. Criteria and indicators of quality and good practice should be established.

Key words: Pediatrics. Primary Health Care. Teamwork. Quality.

\section{Introducción}

En la actualidad el pediatra de Atención Primaria (AP) es el responsable de proporcionar una atención integral y continuada al niño desde el nacimiento hasta los 14 años y su trabajo se desarrolla en el seno de los Equipos Básicos de Salud. El actual modelo de asistencia a la población infantil ha condicionado una mejora en la atención sanitaria a este tramo etario y apostamos por su continuidad, ya que ha demostrado unos excelentes resultados en salud. Los pediatras que trabajamos en el primer nivel asistencial estamos convencidos y de acuerdo con los expertos que los países con una AP de calidad, presentan mejores índices de salud, son más eficientes y reparten de forma más equitativa los recursos sanitarios entre la población' .

En nuestra comunidad autónoma, el "Pacto Andaluz por el desarrollo de estrategias para la Atención Primaria del siglo XXI" (2006-2008) representó para nuestro colectivo el resultado de un in- tenso y sin duda fructífero proceso de negociación desarrollado en los meses previos a su firma. Durante ese tiempo pudimos exponer a los responsables del Servicio Andaluz de Salud la situación de la Pediatría de AP (PAP) y de los profesionales que la desarrollan. Entendíamos que había una serie de aspectos, que debían ser afrontados prioritariamente por el sistema sanitario y que dificultaban las posibilidades de avance y desarrollo. Destacábamos la necesidad de un criterio de ratio pediátrica, la actualización de la cartera de servicios, abordaje del trabajo en equipo, la formación continuada y la rotación del MIR de Pediatría por AP.

El Pacto propició un escenario común para el encuentro con otros profesionales de la AP, como son los colectivos de enfermería y medicina de familia. La administración sanitaria andaluza, a través de este Pacto establecía un marco de actuación sanitaria que posibilitaba una mayor implicación de los profesionales 
en la organización de la asistencia sanitaria y nos situaba como "coprotagonistas" junto a los ciudadanos ${ }^{2}$.

\section{Proyecto de Desarrollo Estratégico} para la Pediatría de Atención Primaria de Andalucía

En este contexto, la Asociación de Pediatras de Atención Primaria de Andalucía (APap-Andalucía) ha realizado un análisis profundo de la situación de la PAP en nuestra comunidad, que se ha concretado en el "Proyecto de Desarrollo Estratégico para la Pediatría de Atención Primaria de Andalucía", un estudio elaborado por la Escuela Andaluza de Salud Pública y promovido por la APapAndalucía. Este trabajo identifica las fortalezas y debilidades internas junto con las amenazas y oportunidades del entorno, buscando el mejor posicionamiento para abordar el futuro de la PAP y el modelo de asistencia a la población infantil ${ }^{3}$.

Sobre la base del análisis de situación previo, nos planteamos la siguiente cuestión: ¿Qué actuaciones se podrían desarrollar con el objetivo de mejorar el posicionamiento y las posibilidades de desarrollo estratégico de la PAP en Andalucía? Las respuestas se agruparon en "Ideas Fuerza", que se clasificaron en 12 categorías que definen las siguientes Lí- neas y Medidas Estratégicas (priorizadas de mayor a menor importancia):

1. Participación e influencia en la Administración: asegurar la "visibilidad" en órganos decisorios identificando el lugar del pediatra en cada línea estratégica de la administración: referentes de Pediatría en Distritos Sanitarios, Consejería de Salud y Servicios Centrales del SAS.

2. Cartera de servicios: definir la Cartera de servicios de la PAP que sirva de guía para solicitar los recursos necesarios para su desarrollo adecuado.

3. Plan de comunicación: aumentar la visibilidad tanto frente a la administración como en nuestro ámbito, así como ante otros colectivos profesionales, la población general.

4. Equipo de atención al niño/trabajo en equipo: implicación del personal de enfermería en la asistencia pediátrica. Potenciar y desarrollar sus perfiles competenciales en este tramo de edad.

5. Impulsar una política adecuada de recursos humanos. Mejora de condiciones laborales: mejorar los contratos laborales que hagan atractivas las plazas de Atención Primaria (estabilidad, horarios que permitan conciliación laboralfamiliar, tamaño de cupos razonables, etc.).

6. Alianzas estratégicas: potenciar la relación con atención especializada, 
otros colectivos y sociedades de AP (Medicina de Familia, Enfermería).

7. Modelo de calidad de la asistencia infantil: establecer el perfil competencial del pediatra de AP actualizado y adecuado a las necesidades de la población, participar en la elaboración de la carrera profesional, requisitos de la agencia de calidad para acreditación de centros, profesionales.

8. Modelo de organización funcional: proponer modelo de organización de la jornada laboral: manejo de pacientes sin cita/urgentes, asegurar la viabilidad de la consulta programada, identificar los problemas en la gestión de las consultas y proponer soluciones (autogestión de la agenda).

9. Investigación: potenciar, incentivar y facilitar la investigación. Visibilizar los logros de la PAP.

10. Formación: aumentar la representación en órganos decisorios del contenido de la especialidad (Comisión Nacional de la especialidad), impulso de la rotación de residente del MIR-Pediatría por AP (adecuación de un programa formativo), asegurar la formación del colectivo, la formación de otros profesionales que forman parte del equipo (Enfermería).

11. Sistemas de registro e información: mejorar/adecuar los sistemas de registro para poder explotar la información recogida, indicadores de calidad, información de actividad, etc.

12. Orientación usuarios/ciudadanos: educación sanitaria para uso responsable de servicios sanitarios. Fomentar los autocuidados.

\section{Las plantillas de pediatras \\ en Andalucía}

Uno de los problemas con que se encuentran los Servicios de Salud actualmente es la falta de profesionales médicos, más acusada en algunas especialidades. La Comisión de Recursos Humanos del Sistema Nacional de Salud hizo público en marzo de 2007 el "Estudio sobre necesidades de especialistas", con proyección de necesidades entre 2006 y 2030. Dicho informe establecía que el SNS tenía un déficit selectivo de profesionales en algunas especialidades y en algunas comunidades autónomas (CCAA). En 2006 eran deficitarias cinco especialidades, entre las que se encontraba la Pediatría.

El Ministerio de Sanidad y Consumo, presentó en un Pleno Extraordinario del Consejo Interterritorial (marzo de 2009), el estudio "Oferta y necesidades de médicos especialistas 2008-2025". Es una versión actualizada y ampliada del primer informe realizado en 2006 , y 
que ahora se constituye en una herramienta metodológica estratégica para mejorar la planificación de los recursos humanos en el sistema sanitario español. En dicho informe, el Ministerio de Sanidad y Consumo propone a las CCAA un decálogo de actuaciones, para una mejor planificación de las necesidades de especialistas en el SNS ${ }^{4}$. Posteriormente, en septiembre de 2009, el Sistema Sanitario Público de Andalucía presenta a los medios el "Estudio de necesidades de especialistas en Andalucía 2009-2039"5.

La PAP en nuestra comunidad tiene en este momento déficit de profesionales, que es más acusado en las zonas rurales, lo que supone en estos momentos una amenaza para el actual modelo de asistencia infantil, relegando a un segundo plano aspectos tan importantes como son la calidad de la asistencia pe- diátrica, las expectativas de las familias y las opiniones de los profesionales sanitarios $^{6}$ (tabla 1).

En el contexto actual de falta de profesionales, la APap-Andalucía se muestra seriamente preocupada porque no se pueda garantizar el acceso de la población menor de 14 años a consultas de Pediatría de AP atendidas por profesionales con formación específica, lo que sin duda debe ser, en estos momentos, una de las prioridades de la Administración sanitaria andaluza (tabla 2).

Es la PAP la que en estos momentos precisa de una atención especial por parte de la Administración sanitaria, debiendo ser el centro de salud el punto de destino preferente para los nuevos pediatras que cada año culminan su formación. El nuevo Plan de Formación de la especialidad contempla la rotación

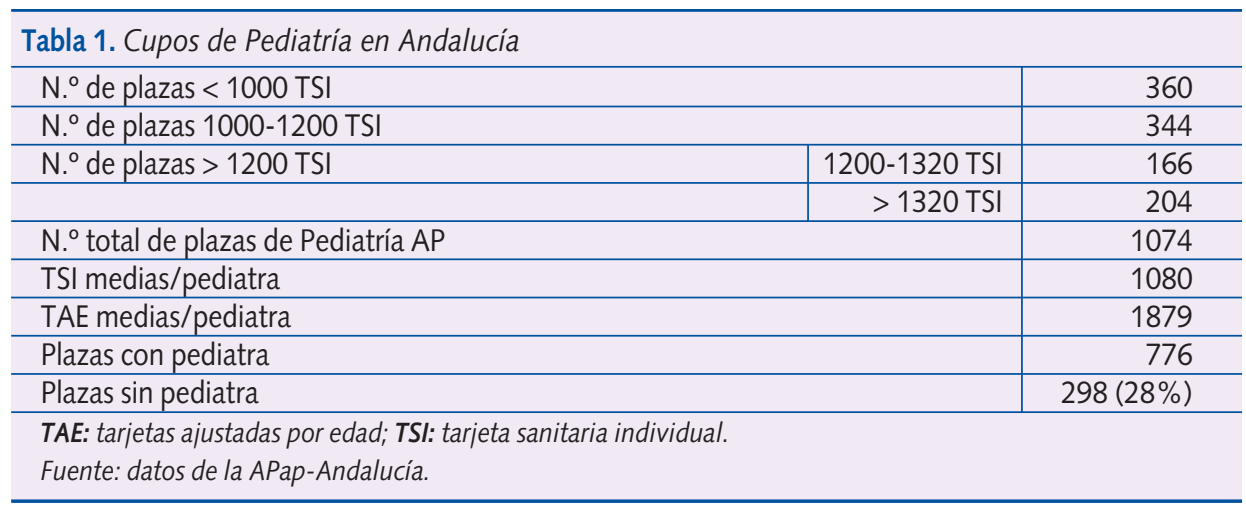


obligatoria por AP, lo que va a permitir que los especialistas en formación conozcan en profundidad nuestro modelo de asistencia a la población infantil (tabla 3).
La falta de pediatras en nuestro ámbito de trabajo, se debe al resultado de la interacción de múltiples circunstancias. La sobrecarga asistencial que los pediatras soportan desde hace tiempo ha

\begin{tabular}{|c|c|c|c|c|}
\hline Provincia & N. ${ }^{\circ}$ de cupos & $\mathrm{N} .^{\circ}$ medio de TSI & Pediatras* & No Pediatras* * \\
\hline Almería & 87 & 1163 & 43 & $44(51 \%)$ \\
\hline Cádiz & 174 & 1129 & 101 & $73(42 \%)$ \\
\hline Córdoba & 100 & 1074 & 72 & $28(28 \%)$ \\
\hline Granada & 117 & 1058 & 93 & $24(21 \%)$ \\
\hline Huelva & 56 & 1099 & 24 & $32(57 \%)$ \\
\hline Jaén & 87 & 966 & 58 & $29(33 \%)$ \\
\hline Málaga & 180 & 1245 & 147 & $33(18 \%)$ \\
\hline Sevilla & 273 & 1036 & 238 & $35(13 \%)$ \\
\hline Total & 1074 & 1080 & 776 & $298(28 \%)$ \\
\hline \multicolumn{5}{|c|}{ TSI: tarjetas sanitarias individuales. } \\
\hline \multicolumn{5}{|c|}{ *Plazas ocupadas por pediatras. } \\
\hline \multicolumn{5}{|c|}{ * *Plazas ocupadas por no pediatras. } \\
\hline \multicolumn{5}{|c|}{ Fuente: datos de la APap-Andalucía. } \\
\hline
\end{tabular}

\begin{tabular}{l|l|c}
\hline \multicolumn{2}{l}{ Tabla 3. Plaza de MIR por provincias } & Plazas MIR \\
\hline Provincia & Centro & 4 \\
\hline Almería & C.H. Torrecárdenas & 3 \\
\hline Cádiz & H. Universitario Puerta del Mar & 1 \\
\hline Puerto Real & H. Universitario de Puerto Real & 1 \\
\hline Jerez de la Frontera & H. de Jerez & 6 \\
\hline Córdoba & H. Universitario Reina Sofía & 5 \\
\hline Granada & H. Universitario San Cecilio & 6 \\
\hline Granada & H. Universitario Virgen de las Nieves & 2 \\
\hline Huelva & H. Juan Ramón Jiménez & 3 \\
\hline Jaén & C.H. Ciudad de Jaén & 12 \\
\hline Málaga & H. Carlos Haya & 1 \\
\hline Marbella & H. Costa del Sol & 1 \\
\hline Sevilla & H. Universitario Ntra. Sra. de Valme & 14 \\
\hline Sevilla & H. Universitario Virgen del Rocio & 6 \\
\hline Sevilla & H. Universitario Virgen Macarena & 65 \\
\hline Total & & \\
\hline $\begin{array}{l}\text { H.: hospital; C.H.: complejo hospitalario; MIR: médico interno residente. } \\
\text { Fuente: Ministerio Sanidad y Consumo. }\end{array}$ \\
\hline
\end{tabular}


provocado cierta resistencia en los nuevos pediatras para trabajar en la AP. El pediatra se ha visto aislado dentro de los Equipos de Atención Primaria y apenas ha recibido apoyo del resto de profesionales del mismo. Las dificultades para sustituir las ausencias (asuntos propios, vacaciones, asistencia a actividades de formación continuada), el sobredimensionamiento de los cupos y la falta de criterios para establecer la ratio adecuada en pediatría, han contribuido a no hacer atractivo el trabajo del pediatra de AP.

La jubilación masiva de los pediatras hospitalarios junto a las referidas condiciones desfavorables de la Pediatría de AP ha condicionado la falta de flujo a primaria de los residentes recién acabados. Por otra parte, el elevado grado de feminización de nuestra profesión obligara a adoptar medidas que flexibilicen la jornada laboral y permitan conciliar la vida laboral y familiar?.

Se habrán de considerar medidas como la captación de pediatras en nuestro entorno (ámbito privado) y fuera de él (otras CCAA y otros países), prolongar la edad de jubilación, mejorar las condiciones laborales con tiempos de consulta adecuados y capacidad de autogestión, implicar a la enfermería en la asistencia infantil, aplicar sin reservas los acuerdos sindicales para las situaciones de acúmulos de cupos, etc.

Las medidas a largo plazo son complejas, pero absolutamente necesarias: la ampliación de números clausus, el incremento del número de plazas MIRPediatría y el aumento de las unidades docentes de Pediatría. Pero son medidas que afectan a diversas administraciones, Salud y Educación, y esta circunstancia dificulta la solución. En este contexto, esperamos que sea la Administración sanitaria andaluza quien asuma la principal responsabilidad y desarrolle un plan de medidas para paliar y afrontar esta realidad.

\section{Los cupos y ratios pediátricos en Andalucía}

La firma del "Pacto Andaluz por el desarrollo de estrategias para la Atención Primaria del siglo XXI" nos permitió alcanzar un compromiso formal entre la Dirección General de Asistencia Sanitaria y la APap-Andalucía, por el que se establecieron los criterios para valorar el crecimiento de la plantilla de pediatras de Equipos Básicos de Atención Primaria (EBAP), durante el periodo 2006-2008. Este hecho ha sido de trascendencia fundamental, ya que nuestro colectivo no había crecido en los últimos años al ritmo que requería la población y las ne- 
cesidades, dificultando las posibilidades de avance y desarrollo de la asistencia infantil. Este compromiso supone para nosotros un paso hacia delante en el establecimiento de una ratio óptima que en estos momentos consideramos debería ser de 1700 tarjetas ajustadas por edad (TAE) (1000 tarjetas sanitarias individuales [TSI])/profesional.

Manifestamos nuestra conformidad con los criterios generales expuestos por la Dirección General de Asistencia Sanitaria, que proponía “desarrollar métodos de ordenación de recursos, ajustados a características poblacionales que incluyan la edad y comorbilidad atendida, el nivel socioeconómico, la dependencia y el modelo organizativo funcional".

La valoración de las necesidades de pediatras se ha realizado traduciéndose las TSI a TAE, para realizar una aproximación más cualitativa a las características de demanda asistencial de la población infantil adscrita al cupo. Para calcular las TAE a partir de las TSI se multiplica por 1,72.

El criterio introducido de "zonas con necesidades de transformación social" (ZNTS), hace referencia a las características de una población en la que se presupone y acepta una mayor necesidad de recursos sanitarios. Las ZNTS están defi- nidas como aquellos espacios urbanos, claramente delimitados, en cuya población concurren situaciones estructurales de pobreza grave y marginación social.

Se considera el Centro de Atención Primaria (CAP) como elemento nuclear o de referencia. Se valora el incremento de plantilla siempre que el cupo medio de TAE pediátricas del Centro supere las siguientes cifras, en tres situaciones distintas:

- Cupo máximo de pediatra itinerante (3 o más CAP): 1720 TAE/1000 TSI. Se ha considerado un incremento de plazas cuando se supera el $10 \%$ de las TAE máximas, es decir, cuando se superan 1892 TAE.

- Cupo máximo en zonas con necesidades de transformación social (ZNTS): 1892 TAE/1100 TSI. Se ha considerado un incremento de plazas cuando se supera el $10 \%$ de las TAE máximas, es decir, cuando se superan 2081 TAE.

- Cupo máximo en el resto de los cupos: 1200 TSI = 2064 TAE. Se ha considerado un incremento de plazas cuando se supera el $10 \%$ de las TAE máximas, es decir, cuando se superan 2270 TAE.

También habrían de considerarse situaciones y ámbitos como son las zonas normalizadas socioeconómicamente, 
pero con gran crecimiento demográfico, las zonas con especial atracción para población inmigrante y el ámbito rural propio de nuestra comunidad autónoma. En nuestra opinión, todos estos aspectos deben tenerse en cuenta de forma diferenciada en la planificación de los recursos sanitarios pediátricos, en aras de evitar inequidades en la prestación de atención sanitaria al ciudadano.

Pensamos que, sobre a base de los criterios referidos, se dispone de un marco de referencia que esperamos sirva a los responsables de los distritos sanitarios para la planificación y el crecimiento de las futuras plantillas de pediatras de EBAP, debiendo considerarse, sin embargo, ritmos de crecimiento superiores a los desarrollados hasta la fecha.

\section{Cartera de servicios}

Destacamos el trabajo desarrollado por el grupo de Cartera de Servicios, que ha realizado una revisión y actualización de la misma según la evidencia científica, y cuya aportación se ha tenido en cuenta e incorporado a la nueva Cartera de Servicios de Atención Primaria del SAS, que fue publicada en junio de 2008 y que incluye importantes novedades en el capítulo de "Atención a la edad pediátrica"8.

La aparición de la Carrera Profesional, cuyos pilares asientan sobre un baremo de méritos bien definido y la consecución de un nivel determinado en la Acreditación de Competencias ha suscitado interés en que los indicadores de calidad se orienten hacia la valoración y consecución de los objetivos de la organización y a que el profesional alcance el máximo nivel en el desarrollo de sus aptitudes y competencias. Intentamos proponer indicadores que cumplan un doble objetivo, por una parte, valorar la calidad de la asistencia prestada y de otra, facilitar el desarrollo profesional.

La PAP debe orientar su práctica hacia la calidad asistencial y centrarla en la consecución de objetivos. Se deben establecer criterios e indicadores de calidad y de buenas prácticas. Estos criterios e indicadores deben basarse en el mapa de competencias del Pediatra de Atención Primaria, en el modelo de carrera profesional en el SAS y en las buenas prácticas que aparecen en la Cartera de Servicios 2008 del SAS 9 .

\section{El papel de la Enfermería en los cuidados a la infancia}

Se identifica la necesidad de potenciar el trabajo en equipo dentro de los Centros de Salud en el que intervenga el personal de Enfermería que asuma actividades asistenciales en este tramo etario. Se trata de impulsar un modelo fun- 
damentado en la cooperación y en la complementariedad. Se hace necesaria la búsqueda de espacios y momentos para el encuentro, huyendo de los compartimentos estancos.

En Andalucía, son pocos los pediatras (menos del 10\%) que cuentan con el apoyo de personal de Enfermería en su práctica diaria. Sería deseable e inaplazable la incorporación de este colectivo profesional a la atención pediátrica a través de un contenido asistencial específico, complementario al del pediatra, de forma que se comparta la asistencia, dotando de actividades propias de la labor de Enfermería (fundamentalmente en la atención de enfermos crónicos, y en las actividades de prevención y promoción de la salud $)^{10}$.

El modelo de Enfermería de familia y comunitaria es coherente, por cuanto que pretende dar respuesta a las necesidades de cuidados del individuo a lo largo de su ciclo vital. Pero el desarrollo que ha tenido apenas ha repercutido o afectado a la Pediatría. esto nos genera muchas dudas para el futuro. Los cuidados de Enfermería han tenido un impacto desigual en lo que respecta a la población infantil en Andalucía.

La infancia es un sector vulnerable de la población y debería ser priorizado, de forma que se aseguraran los cuidados de Enfermería en cualquier situación y momento, tal y como se hace con otros sectores de la población.

Es necesario que se consigan "equipos de profesionales" que compartan una población, en un entorno geográfico delimitado, que la estructura y organización de los centros de salud posibilite espacios temporales y físicos para el trabajo en equipo y que se avance en fórmulas más actuales de gestión de la demanda, que impliquen a cada uno de los miembros del equipo en la respuesta a las demandas de la ciudadanía ${ }^{11}$.

En este marco, la PAP valora positivamente la reciente aprobación del programa formativo de la especialidad de Enfermería Pediátrica, que incluye la rotación específica por AP, lo que sin duda va a suponer una mejora considerable en el perfil competencial de la enfermera en relación con la salud infantil ${ }^{12}$.

\section{La mesa técnica de la Pediatría en Andalucía}

En el mes de noviembre de 2009, la Gerencia del Servicio Andaluz de Salud constituyó una Mesa Técnica con el objetivo de realizar una propuesta de modelo organizativo para la atención pediátrica de Andalucía.

La constitución de esta mesa técnica surge como respuesta a la solicitud de la 
APap-Andalucía, tras el marco del acuerdo con las sociedades científicas que supuso el "Pacto por el desarrollo de la Atención Primaria del Siglo XXI", con el convencimiento de la necesidad de que los profesionales, pediatras de AP y Atención Hospitalaria, participen de la estrategia del sistema sanitario público andaluz aportando su visión como principales protagonistas de la atención sanitaria infantil.

Las propuestas que como colectivo trasladaremos a la Mesa Técnica exigen un esfuerzo de flexibilidad organizativa, precisan invertir en formación, alcanzar un fuerte compromiso de los profesionales, promover cambios organizativos en el seno de los EAP, definir cupos adecuados y dotar de los recursos humanos suficientes ${ }^{11}$.

Desde la APap-Andalucía estamos trabajando por incorporar medidas que mejoren y potencien la asistencia infantil, promoviendo el mejor de los escenarios posibles para dar respuesta a los retos de salud del siglo XXI. Nuestro objetivo prioritario es defender el actual modelo de asistencia pediátrica, basado en la atención directa por pediatras en el primer nivel asistencial.

En algunas CCAA, los Servicios de Salud plantean la necesidad de integración de la Pediatría de AP y Atención Hospitalaria, con una clara intención de resolver solo una parte de los problemas relacionados con la atención infantil, más que con una voluntad de definir y desarrollar las competencias y funciones de cada nivel asistencial a través de la promoción y la coordinación entre niveles, así como la continuidad asistencial'13.

Seguiremos ofreciendo nuestra colaboración a las Instituciones Sanitarias continuando en la línea actual de participación activa, aportando nuestros conocimientos científicos en temas como la actualización de la cartera de servicios, la elaboración y el desarrollo de programas, protocolos y guías de práctica clínica $y$, en general, todas las actuaciones que de una u otra manera repercutan en la salud de la población infantil.

\section{Bibliografía}

1. Buñuel Álvarez JC, García Vera C. ¿Qué profesional médico es el más adecuado para im- partir cuidados en salud a niños en Atención Primaria en países desarrollados? Revisión sistemática. Rev Pediatr Aten Primaria. 2010;12(Supl 18):s9-72. 
2. Pacto Andaluz por el Desarrollo de Estrategias para la Atención Primaria del siglo XXI. [En línea] [consultado el 14/09/2010]. Disponible en www. pediatrasandalucia.org.

3. Valdivia Jiménez C, Palomino Urda N. Proyecto de Desarrollo Estratégico de la Pediatría de AP en Andalucía. Rev Pediatr Aten Primaria. 2008;11(Supl 2):s81-s94.

4. Barber Pérez P, González López-Valcarcel B. Oferta y necesidades de Médicos Especialistas 2008-2025. [En línea] [consultado el 14/09/ 2010]. Disponible en www.msps.es/profesionales.

5. Estudio de necesidades de especialistas en Andalucía 2009-2039. Comunicado de Prensa. Consejería de Salud de la Junta de Andalucía. [En línea] [consultado el 14/09/2010]. Disponible en www.juntadeandalucia.es/salud.

6. Valdivia Jiménez C, Palomino Urda N. Es tiempo de reflexión. Rev Pediatr Aten Primaria. 2008;10:11-5.

7. Ruiz Canela J, Malmierca F. Necesidades de pediatras de Atención Primaria. Rev Pediatria Aten Primaria. 2005;7(Supl 1):s145-55.

8. Cartera de Servicios de Atención Primaria 2008. Consejería de Salud de la Junta de Andalu- cía. [En línea] [consultado el 14/09/2010]. Disponible en www.juntadeandalucia.es/servicioan daluzdesalud/.

9. Navarro Quesada FJ, Gracia Alfonso MA. Atención de calidad en Atención Primaria: propuesta de criterios e indicadores en base al modelo de carrera profesional y el mapa de competencias del pediatra de Atención Primaria. 27. ${ }^{\circ}$ Congreso de la Sociedad Española de Calidad Asistencial. Sevilla, 23/10/2009.

10. Nieto I, Gómez A, Aragón D, Flores L, Vega MA. Enfermería de familia frente a Enfermería de Pediatría. An Esp Pediatr. 2000;53:395-8.

11. Ruiz Canela J, Morell Bernabe J. Un modelo de atención infantojuvenil. Form Act Pediatr Aten Prim. 2009;2(4):181-2.

12. Programa formativo de la especialidad de Enfermería Pediátrica. Orden SAS/1730/2010 de 17 de junio. BOE núm. 157 de 29 de junio. Sec.III: 57251-57276. [En línea] [consultado el 14/09/2010]. Disponible en www.boe.es.

13. Morell Bernabé JJ. Coordinación de recursos en salud infantil. En: AEPap. Curso de Actualización Pediatría 2004. Madrid: Exlibris Ediciones; 2004. p. 111-21. 